\title{
Quantize and Forward Cooperative Communication: Joint Channel and Frequency Offset Estimation
}

\author{
Iancu Avram and Marc Moeneclaey \\ Department of Telecommunications \\ and Information Processing \\ Ghent University \\ 9000 Gent, Belgium \\ Email: firstname.lastname@telin.ugent.be
}

\begin{abstract}
Cooperative communication systems can effectively be used to combat fading. A cooperative protocol that can be used with half-duplex terminals is the quantize and forward (QF) protocol, in which the relay quantizes the information received from the source before forwarding it to the destination. Most studies on the QF protocol are carried out under the assumption of perfect channel state information (CSI) at the destination, which is not often the case in real-life systems. Therefore, in the present contribution, the effect of incomplete CSI is analyzed for flat Rayleigh fading channels with a frequency offset. To limit the complexity of the estimation, the destination terminal assumes that the relay operates in an amplify and forward (AF) mode. By using the expectation maximization (EM) algorithm to refine the initial pilot-based estimates, the resulting error performance can be made very close to that of a system with perfect CSI.
\end{abstract}

Index Terms-Cooperative communication, Quantize-andForward, Iterative Estimation

\section{INTRODUCTION}

Fading is a main limiting factor on the performance of wireless communication systems. In a multipath propagation environment, the incoming signals can interfere both constructively or destructively at the receiving antenna, giving rise to a time-varying signal strength, called fading [1]. When the resulting signal is too weak, an outage event is said to have occurred and reliable information transfer will be impossible [2]. Cooperative communication exploits the broadcast nature of the wireless medium to combat fading [3]. The signal broadcast by the source is received not only by the destination, but also by other terminals nearby. These terminals can be used to introduce spatial diversity by relaying to the destination the information received from the source, creating additional communication channels between source and destination [4], [5]. Different methods can be used for relaying the information from the source to the destination [6]-[8]. A well known relaying strategy is the amplify and forward (AF) protocol, in which the relay amplifies the signal received from the source and forwards it to the destination [9]. While having a seemingly low complexity, the AF protocol, when used with half-duplex devices, requires a large memory at the relay for storing with high precision the received signal awaiting transmission to the destination. This memory requirement is relaxed by the quantize and forward $(\mathrm{QF})$ protocol, in which the received signal is quantized before being stored into mem- ory. This quantization is generally performed on the complex mapper symbols, without taking into account the underlying channel code. By coarsely quantizing the received data, the latter can be stored at the relay awaiting retransmission, with considerably reduced memory requirements as compared to the AF protocol [10].

While the information-theoretical analysis of cooperative systems is of great interest, other aspects also need to be investigated in order to obtain a working implementation. The issue of channel coding for cooperative systems is discussed in [11]. Pilot-based channel estimation is considered in [12], [13] for the AF protocol and theoretical lower bounds (LBs) on the estimation performance have been obtained in [14]. Channel parameter estimation for QF has been investigated in [15], [16] for a protocol in which the relay estimates the source-relay (SR) channel and forwards the estimate to the destination. Another estimation method for the QF protocol, in which all channel parameters are estimated at the destination, is outlined in [17]. In this contribution, the estimation of channel parameters for the QF protocol is analyzed using a more realistic channel model including a carrier frequency offset. All parameters will be estimated at the destination, in order not to increase the complexity of the relay terminal. Due to the frequency offset, the estimation methods from [17] cannot be directly applied here, as this would yield a high increase in computational complexity as explained in subsection II-B. Therefore, a new estimation approach will be outlined in the present contribution.

In section II of this paper the system model is introduced. In section III, the expectation maximization (EM) algorithm [18], [19] is used to derive estimates for all relevant channel parameters, and the initialization of the EM algorithm by means of pilot-based estimates is discussed. The estimation algorithm is validated by means of computer simulations in section IV. Finally, conclusions are drawn in section V.

\section{SySTEM DESCRIPTION}

In this contribution, a cooperative system model consisting of one source, one relay and one destination is considered. All terminals are assumed to be half-duplex devices, meaning that they cannot send and receive information simultaneously. The system architecture is depicted in Fig. 1. In a first timeslot, $K_{d}$ 


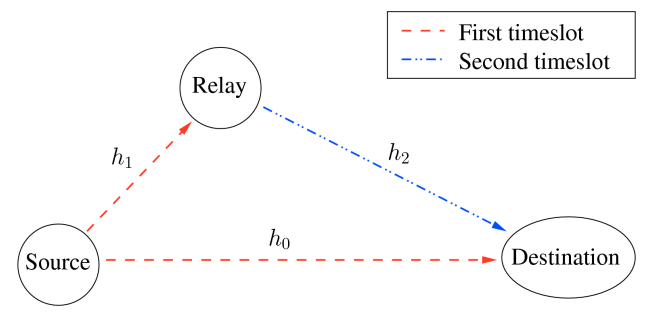

Fig. 1. A relay channel consisting of half-duplex devices.

coded data symbols and $K_{p}$ pilot symbols are broadcast by the source and are received by both the relay and the destination. The $K_{d}$ coded data symbols are obtained by mapping the output of the channel encoder on M-PSK symbols, while the pilot symbols are randomly selected from an M-PSK constellation.

At the relay, the received samples are quantized without knowing the state of the SR channel, as outlined in [10]. Both pilot and data symbols are quantized using the same quantization scheme, yielding a low complexity relay structure. After the quantization operation is completed, the quantized samples are stored in memory awaiting retransmission to the destination in the second timeslot. At the destination, the pilot symbols are used to calculate initial estimates of all the channel parameters involved. Thereafter, the EM algorithm is used to iteratively improve these pilot-based estimates. After convergence of the channel estimates, the channel decoder provides (its decision on) the information bits sent by the source.

\section{A. Communication Channels}

All channels, namely the source-destination (SD), sourcerelay (SR) and relay-destination (RD) channels are considered flat Rayleigh fading channels with additive white Gaussian noise and introducing a carrier frequency offset. The SD, SR and RD channel coefficients are denoted $h_{0}, h_{1}$ and $h_{2}$, respectively, while the $\mathrm{SD}, \mathrm{SR}$ and $\mathrm{RD}$ frequency offsets, normalized to the symbol interval, are denoted $\nu_{0}, \nu_{1}$ and $\nu_{2}$, respectively. Considering this model, the symbol-wise output of the different channels can be written as

$$
r_{i}(k)=h_{i} e^{j 2 \pi k \nu_{i}} c_{i}(k)+n_{i}(k) \quad i \in\{0,1,2\},
$$

with $c_{0}=c_{1}=c_{s}$ being a vector consisting of the symbols sent by the source and $c_{2}=c_{r}$ being a vector consisting of the symbols sent by the relay that correspond to the quantized elements of $\boldsymbol{r}_{1}$. The channel coefficients $h_{i}$ and frequency offsets $\nu_{i}$ are constant during a timeslot. All channel coefficients have a zero mean circular symmetric complex gaussian (ZMCSCG) distribution with variance $N_{h_{i}}=1 / d_{i}{ }^{n}$, with $d_{i}$ the distance between the two terminals involved and $n$ the path loss exponent; this implies a channel gain normalization yielding $\mathrm{E}\left[\left|h_{i}\right|^{2}\right]=1$ for $d_{i}=1$. The value of the frequency offset is uniformly distributed over the frequency uncertainty interval, while the elements of the noise vector $\boldsymbol{n}_{i}$ are ZMCSCG distributed with variance $N_{i}$.
The energies of the symbols sent by the source and the relay are both considered to be equal to $E_{s}$. The latter is related to the energy used to transmit one information bit, denoted $E_{b}$, by the following equation

$$
E_{s}=\frac{K_{d}}{\left(K_{d}+K_{p}\right)} \chi \log _{2}(M) E_{b}
$$

with $\chi$ the code rate used by the channel encoder.

\section{B. Signal Quantization at the Relay}

At the relay, the phase of the received signal is quantized uniformly without taking into account the state of the SR channel. All channel estimation will be performed at the destination. The k-th symbol $c_{r}(k)$ of the vector $\boldsymbol{c}_{r}$ is a quantized version of the k-th element $r_{1}(k)$ of $\boldsymbol{r}_{1}$ :

$$
c_{r}(k)=e^{\frac{j 2 \pi q(k)}{2^{Q}}},
$$

where $Q$ denotes the number of quantization bits, and $q(k)$ is the integer in $\left\{0, \ldots, 2^{Q}-1\right\}$ that satisfies

$$
\frac{\pi}{2^{Q}}(2 q(k)-1) \leq \arg \left(r_{1}(k)\right)<\frac{\pi}{2^{Q}}(2 q(k)+1) .
$$

\section{Likelihood function}

At the destination, the likelihood of the source symbols is needed to decode the received information and to estimate the channel parameters. Introducing $\boldsymbol{x}_{i}=\left(h_{i}, \nu_{i}, N_{i}\right)$, this likelihood can be decomposed as [10]

$$
\begin{aligned}
p\left(\boldsymbol{r}_{0}, \boldsymbol{r}_{2} \mid \boldsymbol{c}_{s}, \boldsymbol{x}_{0}, \boldsymbol{x}_{1}, \boldsymbol{x}_{2}\right)=\prod_{k=1}^{K_{d}} p\left(r_{0}(k) \mid c_{s}(k), \boldsymbol{x}_{0}\right) \\
\sum_{c_{r}(k)} p\left(r_{2}(k) \mid c_{r}(k), \boldsymbol{x}_{2}\right) p\left(c_{r}(k) \mid c_{s}(k), \boldsymbol{x}_{1}\right),
\end{aligned}
$$

with

$$
p\left(r_{i}(k) \mid c_{i}(k), h_{i}, \nu_{i}, N_{i}\right)=\frac{1}{\pi N_{i}} e^{-\frac{\left|r_{i}(k)-h_{i} e^{j 2 \pi k \nu_{i} c_{i}(k)}\right|^{2}}{N_{i}}}
$$

for $i \in\{0,2\}$ and

$$
\begin{aligned}
& p\left(c_{r}(k) \mid c_{s}(k), h_{1}, \nu_{1}, N_{1}\right)= \\
& \quad \int_{\phi_{c_{r}(k)}^{l}}^{\phi_{c_{r}(k)}^{u}} f_{\Theta}\left(\theta-\left(\arg \left(c_{s}(k) h_{1}\right)+2 \pi k \nu_{1}\right) ; \frac{\left|h_{1}\right|^{2}}{N_{1}}\right) \mathrm{d} \theta .
\end{aligned}
$$

The summation over $c_{r}(k)$ in (5) runs over all points of a $2^{Q}$ PSK constellation (see (3)) and the function $f_{\Theta}(\theta ; \gamma)$ is equal to

$$
\begin{aligned}
& f_{\Theta}(\theta ; \gamma)= \\
& \frac{1}{2 \pi}\left[e^{-\gamma}+\sqrt{\pi \gamma} \cos (\theta) e^{-\gamma \sin ^{2}(\theta)} \operatorname{erfc}(-\sqrt{\gamma} \cos (\theta))\right] .
\end{aligned}
$$

The integration in (6) is carried out over the quantization interval (4) that corresponds to the value of $c_{r}(k)$ in the summation (5). The corresponding integral must be computed for all $2^{Q}$ values of $c_{r}(k)$ and all $M$ values for $c_{s}(k)$. Due to the presence of the frequency offset $\nu_{1}$ in the argument of $f($. in (6), the integral for given $c_{r}(k), c_{s}(k)$ and $\boldsymbol{x}_{1}$ depends on the symbol index $k$ within a frame. Therefore, the evaluation of 
the likelihood function (5) for given channel parameters would require that the integral in (6) be computed numerically (for all $2^{Q}$ values of $c_{r}(k)$ and all $M$ values for $c_{s}(k)$ ) for each of the $K_{p}+K_{d}$ symbol indices.

In [17], where we considered the same problem in the absence of frequency offsets, estimation and detection algorithms have been derived based on (5). In the case of zero frequency offsets, the integral in (6) for given $c_{r}(k), c_{s}(k)$ and $\boldsymbol{x}_{1}$ becomes independent of the symbol index $k$, yielding an acceptable computational complexity. However, straightforward extension of the algorithms from [17] to accommodate nonzero frequency offsets would drastically increase the computational complexity, as the integral (6) becomes a function of the symbol index $\mathrm{k}$.

In order to avoid the high computational complexity associated with the evaluation of (5) for nonzero frequency offset, we will simplify the problem by considering a destination node that (falsely) assumes that the relay performes an amplify and forward operation on the samples received from the source ${ }^{1}$. This assumption involves replacing (3) with

$$
c_{r}(k)=r_{1}(k) /\left|h_{1}\right|
$$

Using (8) in (1), the latter can be written as

$$
\begin{aligned}
r_{2}(k) & =h_{2} e^{j 2 \pi k \nu_{2}}\left(r_{1}(k) /\left|h_{1}\right|\right)+n_{2}(k) \\
& =h_{3} e^{j 2 \pi k \nu_{3}} \boldsymbol{c}_{s}+n_{3}(k),
\end{aligned}
$$

with

$$
\begin{aligned}
& n_{3}(k)=h_{2} e^{j 2 \pi k \nu_{2}}\left(\frac{n_{1}(k)}{\left|h_{1}\right|}\right)+n_{2}(k) \\
& h_{3}=\frac{h_{1} h_{2}}{\left|h_{1}\right|} \quad \nu_{3}=\nu_{1}+\nu_{2} .
\end{aligned}
$$

Introducing $\boldsymbol{x}_{3}=\left(h_{3}, \nu_{3}, N_{3}\right)$, the corresponding likelihood function associated with the observation $r_{2}(k)$ can be written as

$$
p\left(r_{2}(k) \mid c_{s}(k), \boldsymbol{x}_{\mathbf{3}}\right)=\frac{1}{\pi N_{3}} e^{-\frac{\left|r_{2}(k)-h_{3} e^{j 2 \pi k \nu_{3} c_{s}(k)}\right|^{2}}{N_{3}}},
$$

with $N_{3}$ the variance of the elements in $\boldsymbol{n}_{3}$.

\section{Channel estimation}

The symbol likelihoods obtained in section II-C contain the channel parameters $h_{0}, \nu_{0}$ and $N_{0}$ corresponding to the direct channel and the parameters $h_{3}, \nu_{3}$ and $N_{3}$ corresponding to the relay channel. As these parameters are not a-priori known to the destination, they will need to be estimated before the received information can be decoded. This estimation uses in a first stage the pilot symbols sent by the source. The quality of these pilot-based estimates improves as the number of pilot symbols is increased. However, the addition of pilot symbols decreases the symbol energy as shown by (2). Therefore, the number of pilot symbols should be limited. These seemingly contradictory demands can be reconciled

\footnotetext{
${ }^{1}$ The performance results from section IV correspond to a QF relay, with the destination falsely assuming an AF relay.
}

using the EM algorithm. The EM algorithm is an iterative algorithm that also uses the unknown data symbols in the estimation process. In doing so, the estimates can be improved without the addition of supplementary pilot symbols.

\section{A. Pilot-based estimates}

In this subsection, the pilot symbols sent by the source are used to obtain pilot-based estimates of the different channel parameters involved. Later on, these estimates will be refined using the EM algorithm. A maximum likelihood (ML) estimate of the different channel parameters is found by solving the following equation

$$
\left(\hat{\boldsymbol{x}_{0}}, \hat{\boldsymbol{x}_{3}}\right)=\arg \max _{\left(\boldsymbol{x}_{0}, \boldsymbol{x}_{3}\right)} p\left(\boldsymbol{r}_{0: p}, \boldsymbol{r}_{2: p} \mid \boldsymbol{c}_{s: p}, \boldsymbol{x}_{0}, \boldsymbol{x}_{3}\right),
$$

with the subscript $: p$ used to denote the part of a vector that corresponds to the pilot symbol positions. Using (5), (10) can be written as

$$
\left(\hat{\boldsymbol{x}}_{i}\right)=\arg \max _{\boldsymbol{x}_{i}} p\left(\boldsymbol{r}_{i: p} \mid \boldsymbol{c}_{s: p}, \boldsymbol{x}_{i}\right) \quad i \in\{0,3\},
$$

with the convenient notation of $\boldsymbol{r}_{3}=\boldsymbol{r}_{2}$ and $\boldsymbol{c}_{3}=\boldsymbol{c}_{2}$. Using (9), (11) can be written as

$$
\begin{aligned}
\left(\hat{\boldsymbol{x}}_{i}\right)=\arg \min _{\boldsymbol{x}_{i}} & K_{p} \ln \left(\pi N_{i}\right) \\
& +\sum_{k=1}^{K_{p}} \frac{\left|r_{i: p}(k)-h_{i} e^{j 2 \pi k \nu_{i}} c_{s: p}(k)\right|^{2}}{N_{i}} .
\end{aligned}
$$

By inserting a given trial value $\tilde{\nu_{i}}$ and $\tilde{h_{i}}$ in (12), the value of the noise variance that satisfies (12) is found to be

$$
\hat{N}_{i}=\frac{\sum_{k=1}^{K_{p}}\left|r_{i: p}(k)-\tilde{h_{i}} e^{j 2 \pi k \tilde{\nu_{i}}} c_{s: p}(k)\right|^{2}}{K_{p}} .
$$

Using this value in (12) yields

$$
\left(\hat{h_{i}}, \hat{\nu_{i}}\right)=\arg \min _{\left(h_{i}, \nu_{i}\right)} \sum_{k=1}^{K_{p}}\left|r_{i: p}(k)-h_{i} e^{j 2 \pi k \nu_{i}} c_{s: p}(k)\right|^{2} .
$$

A computationally efficient solution to this problem has been obtained in [20] for MIMO systems. Applying this solution to a SISO system yields the following estimates for the frequency offset and the channel coefficient

$$
\begin{gathered}
\hat{\nu_{i}}=\frac{\sum_{m=1}^{K_{p}} m\left|R_{i: p}(m)\right| \arg \left(R_{i: p}(m)\right)}{2 \pi \sum_{m=1}^{K_{p}} m^{2}\left|R_{i: p}(m)\right|} \\
\hat{h_{i}}=\frac{\sum_{k=1}^{K_{p}} r_{i: p}(k) c_{s: p}(k)^{*} e^{-j 2 \pi k \nu_{i}}}{K_{p} E_{s}}
\end{gathered}
$$

with

$$
R_{i: p}(m)=\frac{\sum_{k=1}^{K_{p}} r_{i: p}(k-m)^{*} r_{i: p}(k) c_{s: p}(k)^{*} c_{s: p}(k-m)}{K_{p} E_{s}} .
$$




\section{B. EM estimation}

The EM algorithm allows calculating an estimate of unknown parameters while also utilizing the (unknown) data symbols, named nuisance parameters. In the case at hand, the data symbols sent by the source are considered as nuisance parameters. The EM algorithm iterates between an expectation and maximization step. In the expectation step, the estimates from the previous iteration are used to compute the a-posteriori expectation of the data symbols. In the maximization step, these expectations are used to update the channel parameter estimates. Introducing $\boldsymbol{r}_{d}=\left(\boldsymbol{r}_{0}, \boldsymbol{r}_{2}\right)$, the expectation step consists of calculating the following function

$$
\begin{aligned}
& Q\left(\boldsymbol{x}_{0}, \boldsymbol{x}_{3}, \hat{\boldsymbol{x}}_{0}{ }^{(j-1)}, \hat{\boldsymbol{x}}_{3}{ }^{(j-1)}\right)= \\
& \mathrm{E}_{\boldsymbol{c}_{s}}\left[\ln p\left(\boldsymbol{r}_{d} \mid \boldsymbol{c}_{s}, \boldsymbol{x}_{0}, \boldsymbol{x}_{3}\right) \mid \boldsymbol{r}_{d}, \hat{\boldsymbol{x}}_{0}{ }^{(j-1)}, \hat{\boldsymbol{x}}_{3}{ }^{(j-1)}\right] .
\end{aligned}
$$

The maximization step consists of finding the value of $\boldsymbol{h}, \boldsymbol{f}$ and $N$ that maximizes the Q function from (15), i.e

$$
\left(\hat{\boldsymbol{x}}_{0}{ }^{(j)}, \hat{\boldsymbol{x}}_{3}{ }^{(j)}\right)=\arg \max _{\left(\boldsymbol{x}_{0}, \boldsymbol{x}_{3}\right)} Q\left(\boldsymbol{x}_{0}, \boldsymbol{x}_{3}, \hat{\boldsymbol{x}}_{0}{ }^{(j-1)}, \hat{\boldsymbol{x}}_{3}{ }^{(j-1)}\right) \text {. }
$$

The estimates $\hat{\boldsymbol{x}}_{0}{ }^{(0)}$ and $\hat{\boldsymbol{x}}_{3}{ }^{(0)}$, used to initialize the iterative process, correspond to the pilot-based estimates obtained in the previous subsection. The same techniques used to obtain the pilot-based estimates can be applied to solve the maximization problem from (16), yielding the following estimates

$$
\begin{aligned}
{\hat{\nu_{i}}}^{(j)} & =\frac{\sum_{m=1}^{K_{p}+K_{d}} m\left|R_{i}^{(j)}(m)\right| \arg \left(R_{i}^{(j)}(m)\right)}{2 \pi \sum_{m=1}^{K_{p}+K_{D}} m^{2}\left|R_{i}^{(j)}(m)\right|} \\
{\hat{h_{i}}}^{(j)} & =\frac{\sum_{k=1}^{K_{p}+K_{d}} r_{i}(k) u_{s}^{(j)}(k)^{*} e^{-j 2 \pi k \hat{\nu}_{i}}{ }^{(j)}}{E_{s}\left(K_{p}+K_{d}\right)} \\
\hat{N}_{i}{ }^{(j)} & =\frac{\sum_{k=1}^{K_{p}+K_{d}}\left|r_{i}(k)-{\hat{h_{i}}}^{(j)} e^{j 2 \pi k \hat{\nu}_{i}{ }^{(j)}} u_{s}^{(j)}(k)\right|^{2}}{K_{p}+K_{d}},
\end{aligned}
$$

with $R_{i}^{(j)}$ defined similarly to (14), with the vector $\boldsymbol{c}_{s: p}$ replaced by $\boldsymbol{u}_{s}^{(j)}$. The vector $\boldsymbol{u}_{s}^{(j)}$ denotes the a posteriori expectation (conditioned on $\boldsymbol{r}_{d}, \hat{\boldsymbol{x}}_{0}{ }^{(j-1)}$ and $\hat{\boldsymbol{x}}_{3}{ }^{(j-1)}$ ) of the symbol vector $\boldsymbol{c}_{s}$. The components of $\boldsymbol{u}_{s}$ that correspond to the pilot symbols are equal to these pilot symbols, while the components of $\boldsymbol{u}_{s}$ that correspond to the data symbols are defined by

$$
u_{s}(k)=\sum_{c_{s}(k)} c_{s}(k) p\left(c_{s}(k) \mid \boldsymbol{r}_{d}, \hat{\boldsymbol{x}}_{0}{ }^{(j-1)}, \hat{\boldsymbol{x}}_{3}{ }^{(j-1)}\right),
$$

where summation run over all values that $c_{s}(k)$ can adopt. The marginal a posteriori probabilities of the data symbols $c_{s}(k)$ can be calculated by the decoder at the destination [21].

\section{NUMERICAL RESULTS}

In this section, computer simulations are used to analyze the implications of the AF approximation from subsection II-C and the performance of the estimates discussed in section III. First, the codeword error rate (WER) performance is studied,

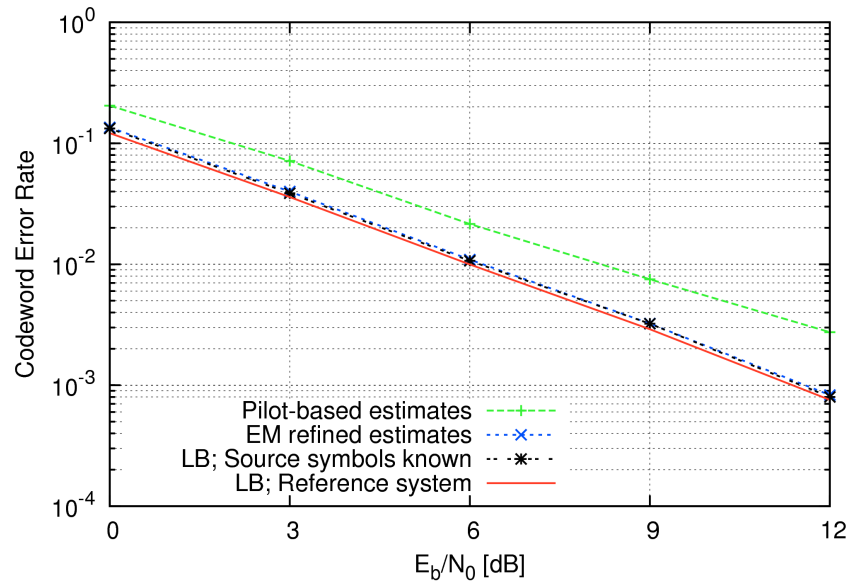

Fig. 2. WER performance of a BPSK-mapped system and 2 bit quantization at the relay.

whereafter the MSE performance is discussed to provide more insight into the estimation process. We consider a source that encodes frames of 1024 information bits by means of a $(1,13 / 15)_{8}$ RSCC turbo code [22] that is punctured to a rate of $2 / 3$. After encoding, the coded bits are mapped on $\mathrm{M}$ PSK symbols. Depending on the constellation size, $\log _{2}(M)$ codewords are grouped into one frame, yielding frames of 1536 data symbols, irrespective of $M$. The normalized carrier frequency offsets $\nu_{i}, i \in\{0,1,2\}$, are uniformly distributed within the interval $\left[-\frac{1}{4\left(K_{p}+K_{d}\right)}, \frac{1}{4\left(K_{p}+K_{d}\right)}\right]$, yielding a maximum rotation of $\mp \pi / 2$ within one frame.

To each frame are added only 12 pilot symbols, which will be used at the destination to calculate the pilotbased estimates; the power loss corresponding to (2) equals $10 \log _{10}(1548 / 1536)=0.034 d B$. The relay is located halfway between source and destination. The path loss exponent equals 4 and the distance between source and destination is considered unity. For a given $E_{b} / N_{0}$ ratio, the energy of the symbols transmitted by the source and relay is computed using (2). All noise variances are assumed to be equal $\left(N_{0}=N_{1}=N_{2}\right)$. Hence, $E_{b} / N_{0}$ is representative of the SNR of the SD link. When using the EM algorithm, the EM iterations and turbo decoding iterations are merged as explained in [23].

\section{A. WER performance}

Fig. 2 shows the WER performance of a system in which only pilot-based estimates are used and that of a system in which these pilot-based estimates are refined using the EM algorithm. Both systems use BPSK-mapping at the source and a 2 bit quantization scheme (yielding a QPSK constellation for $\left.c_{r}(k)\right)$ at the relay. The figure also shows 2 lower bounds (LBs) on the WER performance. The first LB, referred to as 'reference system', corresponds to a system in which all channel parameters are known to the destination and in which the detection is based on the likelihoods that are computed by numerically integrating (6) (and not using the AF approximation). A tighter LB, referred to as 'source symbols known', 


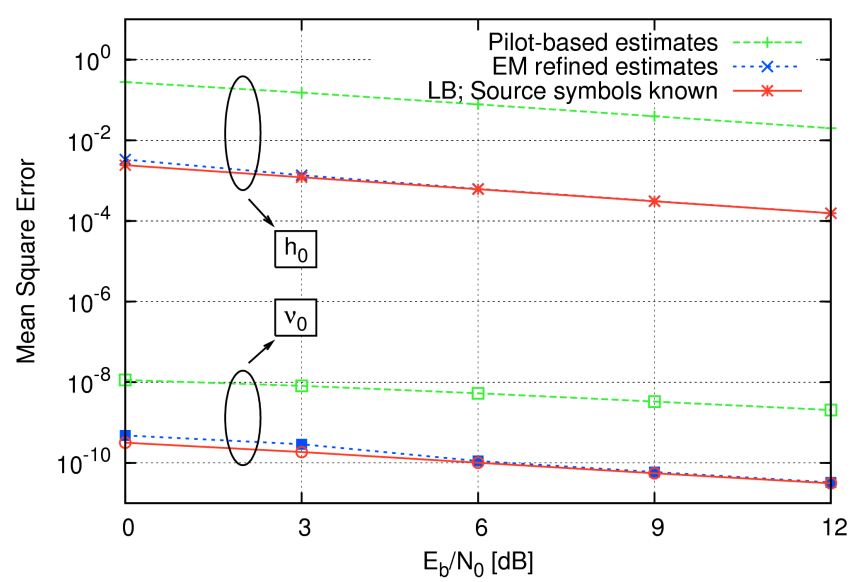

Fig. 3. MSE of $\hat{h_{0}}$ and $\hat{\nu_{0}}$ as function of the $E_{b} / N_{0}$ ratio.

corresponds to a system in which the symbols sent by the source are considered to be known to the destination when calculating the channel estimates (i.e., we take $\boldsymbol{u}_{s}=\boldsymbol{c}_{s}$ in (17)) and in which the symbol likelihoods needed to decode the received infomation are calculated using the computationally much less demanding $\mathrm{AF}$ approximation. Both LBs also take into account the $0.034 d B$ energy degradation caused by the addition of pilot symbols.

As can be seen on Fig. 2, the system using only pilotbased estimates achieves a poor WER performance compared to the reference system. This WER performance is greatly improved by the use of the EM algorithm, achieving a WER performance that is close to that of the reference system. The WER performance associated with the EM algorithm coincides with that of the second LB, suggesting that the calculated aposteriori symbol expectations, denoted by $\boldsymbol{u}_{s}$, are close to the actual symbols sent by the source. The small difference in WER performance between the second LB and the reference system confirms the usefulness of the AF approximation.

\section{B. MSE performance}

In this subsection the MSE performance of the different estimation methods is studied. To better understand the estimation of the channel parameters $\boldsymbol{x}_{3}$, the channel coefficient $h_{i}$ will be represented as an amplitude component and a phase component, i.e. $h_{i}=A_{i} e^{\phi_{i}}, i \in\{1,2,3\}$. The estimate of $A_{3}$, denoted as $\hat{A_{3}}$, is set equal to $\left|\hat{h_{3}}\right|$, while the phase estimate $\hat{\phi_{3}}$ is set equal to $\arg \left(\hat{h_{3}}\right)$. Further, we introduce $\theta_{i}(k)=\phi_{i}+2 \pi \nu_{i} k$ for $i \in\{1,2,3\}$ and $\hat{\theta}_{3}(k)=\hat{\phi}_{3}+2 \pi \hat{\nu}_{3} k$.

Fig. 3 shows the MSE performance of the SD channel estimates $\hat{h_{0}}$ and $\hat{\nu_{0}}$, while Fig. 4, 5 and 6 show the MSE performance of $\hat{A_{3}}, \hat{\phi_{3}}$ and $\hat{\nu_{3}}$, respectively. In all four figures BPSK-mapping is used at the source and the relay quantizes the received information using 2 and 4 bits. A LB on the MSE performance is also shown, corresponding to the 'source symbols known' situation described in the previous subsection. As shown in Fig. 3, the MSE of $\hat{h_{0}}$ and $\hat{\nu_{0}}$ decreases with increasing SNR and approaches the LB for moderate and high SNR. The same applies to the MSE of $\hat{A}_{3}$, however with

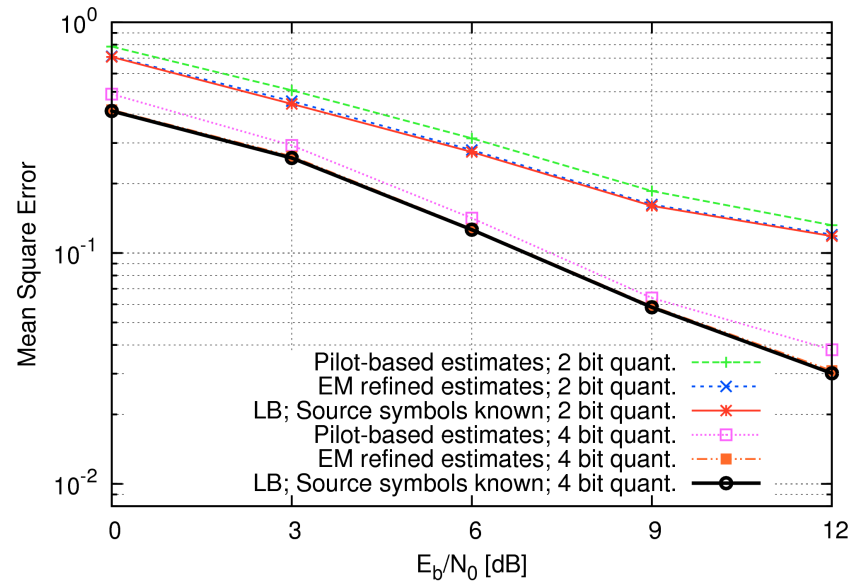

Fig. 4. MSE of $\hat{A_{3}}$ as function of the $E_{b} / N_{0}$ ratio and the number of quantization bits used at the relay.

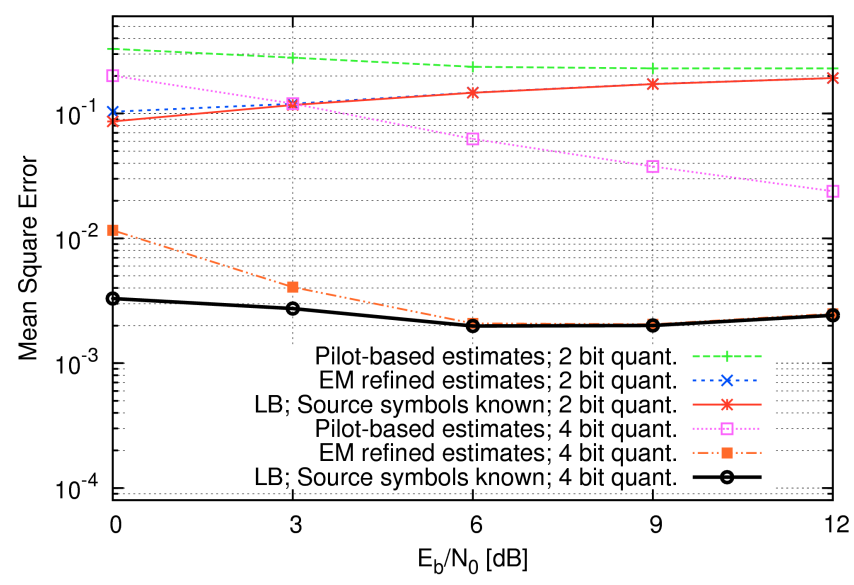

Fig. 5. MSE of $\hat{\phi_{3}}$ as function of the $E_{b} / N_{0}$ ratio and the number of quantization bits used at the relay.

smaller differences between the pilot-based estimates and the EM refined ones. The MSE of $\hat{A}_{3}$ also decreases when the number of quantization bits is increased, as a finer quantization scheme allows for a more accurate estimation.

Studying the MSE of $\hat{\phi_{3}}$ and $\hat{\nu_{3}}$, one observes that the MSE of the EM refined estimates still approaches the LB, but the LB at high SNR shows an error floor that is close to that of a system using only pilot-based estimates. This error floor can be understood by studying the signal received from the relay in the absence of noise. Under this assumption, these samples can be written as

$$
\left.r_{2}(k)=A_{2} c_{s}(k) e^{j\left(\theta_{2}(k)+\operatorname{round}\left(\theta_{1}(k)\right)\right.}\left(\frac{2 \pi}{2 Q}\right)\right),
$$

where round $(.)_{\left(\frac{2 \pi}{2}\right)}$ denotes rounding to the nearest integer multiple of $\frac{2 \pi}{2 Q}$. Noting that the receiver falsely assumes that

$$
r_{2}(k)=A_{2} c_{s}(k) e^{j\left(\theta_{2}(k)+\theta_{1}(k)\right)}=A_{3} c_{s}(k) e^{j \theta_{3}(k)},
$$

comparison of (18) and (19) indicates that because of the quantization at the relay, some of the information contained in 


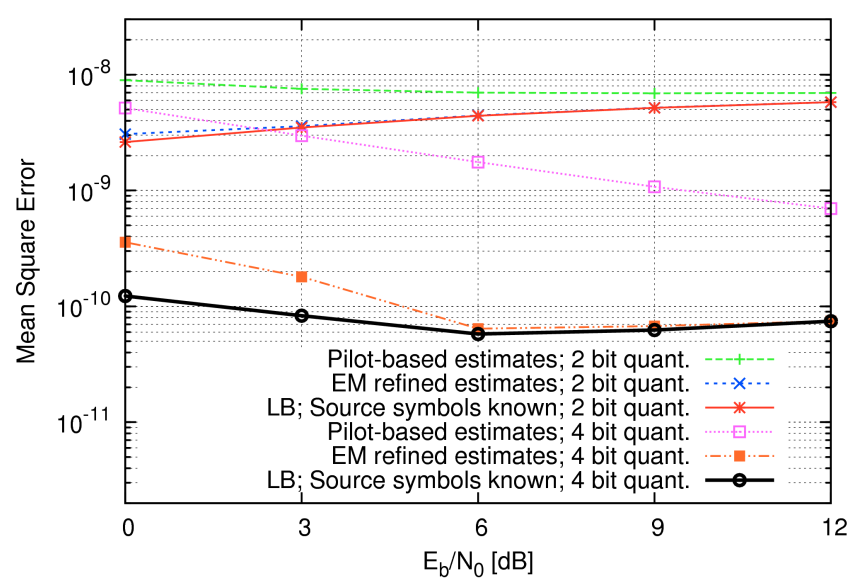

Fig. 6. MSE of $\hat{\nu_{3}}$ as function of the $E_{b} / N_{0}$ ratio and the number of quantization bits used at the relay.

$\theta_{1}(k)$ is lost due to the rounding operation, so that $\theta_{3}(k)$ (or, equivalently, $\phi_{3}$ and $\nu_{3}$ ) cannot be recovered at the destination. Actually, because of (18), $\hat{\theta}_{3}(k)$ is essentially a linear (in the time variable $\mathrm{k})$ fit to $\left.\theta_{2}(k)+\operatorname{round}\left(\theta_{1}(k)\right)_{\left(\frac{2 \pi}{2} Q\right.}\right)$, hence $\hat{\theta}_{3}(k)$ is an appropriate angle to be used for derotating $r_{2}(k)$ from (18) for the purpose of detection. Therefore, the fact that $\hat{\theta}_{3}(k)$ is a poor estimate of $\theta_{3}(k)$ does not affect the reliability of the detector. We see from Figs. 5 and 6 that for some parameter settings the estimation accuracy improves with decreasing SNR; this is because the noise-induced jumps to adjacent quantization levels provide additional information about $\theta_{1}(k)$.

\section{Conclusions}

In this contribution, channel parameter estimation in the presence of a frequency offset is discussed for a QF cooperative protocol. The estimation is carried out under the (false) assumption that the relay performs an AF operation on the received samples. Using this approximation, the computation of the symbol likelihoods at the destination and the estimation process are greatly simplified. By estimating all channel parameters at the destination, the relay complexity is also kept low, making the obtained algorithms suitable for the use in sensor networks and other low complexity relaying applications.

While keeping the complexity at the relay and destination terminals low, the proposed estimation method exhibits a good WER performance as shown in section IV. By refining the pilot-based estimates using a code-aided EM approach, the loss in WER performance compared to a system with known channel parameters can be made very small. Finally, by studying the MSE values, more insight is gained in the process of estimating channel parameters using quantized data.

\section{ACKNOWLEDGMENT}

The authors wish to acknowledge the Agency for Innovation by Science and Technology Flanders (IWT) that motivated this work.

\section{REFERENCES}

[1] B. Sklar Rayleigh Fading Channels in Mobile Digital Communication Systems Part I: Characterization. IEEE Communications Magazine, vol. 35, no. 7, pp. 90-100, Jul. 1997

[2] T. M. Cover and J. A. Thomas Elements of Information Theory. Wiley, New York, 1991

[3] A. Sendonaris, E. Erkip and B. Aazhang User cooperation diversity part I:system Description. IEEE Trans. on Commun., vol. 51, no. 11, pp. 1927-1938, Nov. 2003

[4] T. M. Cover and A. El Gamal Capacity theorems for the relay channel. IEEE Trans. Inf. Theory, vol. 25, no. 5, pp. 572-584, Sep. 1979

[5] A. El Gamal, M. Mohseni and S. Zahedi Bounds on capacity and minimum energy-per-bit for AWGN relay channels. IEEE Trans. Inf. Theory, vol. 52, no. 4, pp. 1545-1561, Apr. 2006

[6] J. N. Laneman, D. N. C. Tse and G. W. Wornell Cooperative diversity in wireless networks: efficient protocols and outage behavior. IEEE Trans. Inf. Theory., vol. 50, no. 12, pp. 3062-3080, Dec. 2004

[7] S. Lee, W. Su, S. Batalama and J. D. Matyjas Cooperative Decode-andForward ARQ Relaying Performance Analysis and Power Optimization. IEEE Trans. on Wireless Commun., vol. 9, no. 8, pp. 2632-2642, Aug. 2010

[8] T. E. Hunter, S. Sanayei and A. Nosratinia Outage Analysis of Coded Cooperation. IEEE Trans. Inf. Theory., vol. 52, no. 2, pp. 375-391, Feb. 2006

[9] J. N. Laneman Cooperative Diversity in Wireless networks: Algorithms and Architectures. Massachusetts Institute of Technology, 2002

[10] M. Souryal and H. You Quantize-and-Forward Relaying with M-ary Phase Shift Keying. IEEE Wireless Communications and Networking Conference 2008, pp. 42-47

[11] A. Chakrabarti, A. de Baynast, A. Sabharwal and B. Aazhang LDPC code-design for the relay channel. IEEE J. Select. Areas Commun. Special Issue on Cooperative Communications and Networking, vol. 25, no. 2, pp. 280-291, Feb. 2007

[12] B. Gedik and M. Uysal Impact of Imperfect Channel Estimation on the Performance of Amplify-and-Forward Relaying. IEEE Trans. on Wireless Commun., vol. 8, no. 3, p. 1468-1479, Mar. 2009

[13] C. S. Patel and G. L. Stüber Channel Estimation for Amplify and Forward Relay Based Cooperation Diversity Systems. IEEE Trans. on Wireless Commun., vol. 6, no. 6, pp. 2348-2356, Jun. 2007

[14] N. Aerts, I. Avram and M. Moeneclaey The Cramer-Rao Bound for channel estimation in block fading Amplify-and-Forward relaying networks. Spread Spectrum Techniques and Applications (ISITA), 2010 IEEE 11th International Symposium on, pp. 12-16

[15] I. Avram, N. Aerts and M. Moeneclaey A Novel Quantize-and-Forward Cooperative System: Channel Parameter Estimation Techniques. Future Network \& Mobile Summit 2010, Florence, Italy, 16-18 Jun. 2010

[16] I. Avram, N. Aerts, D. Duyck, and M. Moeneclaey A Novel Quantizeand-Forward Cooperative System: Channel Estimation and M-PSK Detection Performance. EURASIP Journal on Wireless Communications and Networking, vol. 2010, Article ID 415438, 11 pages

[17] I. Avram, N. Aerts, H. Bruneel and M. Moeneclaey Quantize and Forward Cooperative Communication: Channel Parameter Estimation. IEEE Trans. on Wireless Commun., vol. 11, no. 3, pp. 1167-1179, Mar. 2012

[18] A. Dempster, N. Laird and D.B. Rubin Maximum Likelihood From Incomplete Data Via the EM Algorithm. J. Royal Statistical Soc., vol. 39 , no. 1 , pp. 1-38, 1977

[19] H. Wymeersch, F. Simoens and M. Moeneclaey Code-aided Joint Channel Estimation and Frame Synchronization for MIMO Systems. IEEE 5th workshop on Signal Processing Advances in Wireless Communications, 2004 , pp. $82-86$

[20] F. Simoens and M. Moeneclaey Reduced Complexity Data-Aided and Code-Aided Frequency Offset Estimation for Flat-Fading MIMO Channels. IEEE Trans. on Wireless Commun., vol. 5, no. 6, pp. 15581567, Jun. 2006

[21] H. Wymeersch Iterative Receiver Design. Cambridge University Press, 2007

[22] S. Lin and D. Costello Error Control Coding. Pearson Education Inc., second edition, 2004

[23] N. Noels, C. Herzet, A. Dejonghe, V. Lottici, H. Steendam, M. Moeneclaey, M Luise and L. Vandendorpe Turbo-synchronization: an EM algorithm approach. Proc. IEEE ICC, Anchorage, May 2003 